Bundesgesundheitsbl - Gesundheitsforsch Gesundheitsschutz 2005 • 48:963-970

DOI 10.1007/s00103-005-1127-1

๑) Springer Medizin Verlag 2005

O. Hamouda · Robert Koch-Institut, Berlin

\title{
Die Rolle des Bundes bei der Verhütung und Bekämpfung von Infektions- krankheiten
}

\section{Strukturen, Zuständigkeiten und Aufgaben - eine Übersicht}

sel zur Verbesserung des Gesundheitszustands der Bevölkerung und zur Senkung der Kosten im Gesundheitssektor. Sie stellen daher eine wichtige Säule im Gesundheitswesen dar. Die Prävention von Infektionskrankheiten und der Infektionsschutz sind dabei prioritäre Aufgaben bei der Fortentwicklung eines leistungsfähigen Gesundheitswesens in Deutschland.

Das Infektionsschutzgesetz (IfSG) trat am 1.1.2001 in Kraft und stellte das System der meldepflichtigen Krankheiten in Deutschland auf eine neue Basis [1]. Das IfSG regelt, welche Krankheiten bei Verdacht, Erkrankung oder Tod und welche labordiagnostischen Nachweise von Erregern meldepflichtig sind. Weiterhin legt das Gesetz fest, welche Angaben von den Meldepflichtigen zu machen und welche vom Gesundheitsamt weiter zu übermitteln sind $[2,3]$. Mit der Einführung des IfSG wurden in Deutschland Falldefinitionen zur routinemäßigen Übermittlung der meldepflichtigen übertragbaren Krankheiten eingeführt [4]. Darüber hinaus soll mit dem IfSG auch die Stärkung der Eigenverantwortung von Personen in infektionsgefährdeten Bereichen gefördert werden.

Der gezielte Einsatz wirksamer Schutzimpfungen, die Sicherung hoher hygienischer Standards in der Patientenversorgung und die Entwicklung wirksamer an- timikrobieller Wirkstoffe sind darüber hinaus wichtige Ziele und Maßnahmen des Infektionsschutzes.

Aber auch die gesundheitsbezogene Selbsthilfe leistet wichtige Beiträge zur Ergänzung der Versorgung im Gesundheitswesen. Selbsthilfegruppen und -organisationen helfen vor allem chronisch Kranken und behinderten Menschen, durch Information und gegenseitige Unterstützung das Leben mit einer Krankheit oder Behinderung besser zu bewältigen.

Ein weiterer Teil des Gesundheitsschutzes ist der Schutz der Patienten vor Gefahren, die von Arzneimitteln und Medizinprodukten ausgehen können. Er wird durch die qualitativ hochwertige Zulassung von Arzneimitteln durch kompetente Zulassungsbehörden insbesondere durch das Bundesinstitut für Arzneimittel und Medizinprodukte (BfArM), das Paul-Ehrlich-Institut (PEI) sowie das Bundesamt für gesundheitlichen Verbraucherschutz und Lebensmittelsicherheit (BVL, Zulassung und Registrierung von Tierarzneimitteln) sichergestellt.

Gesundheit hängt wesentlich auch von äußeren Einflüssen wie den Umweltbedingungen ab. Ein präventionsorientierter $\mathrm{Ge}$ sundheitsschutz erfordert daher einen ressortübergreifenden Politikansatz, der frühzeitiges Erkennen von Gesundheitsrisiken und ein entsprechendes Reagieren ermög- 
licht. Gesundheitsaspekte werden hierbei verstärkt in die nationale und internationale Umweltpolitik eingebracht.

Im Rahmen des Gesundheitsschutzes ist zudem die Krisenreaktionsfähigkeit des Bundes bei bioterroristischen Gefahrenlagen sicherzustellen. Hierfür werden bestehende Konzepte zur Vorsorge und zum Krisenmanagement bei terroristischen Anschlägen mit biologischen Wirkstoffen weiterentwickelt.

Die Bewältigung der genannten Aufgaben erfordert das enge Zusammenwirken einer Reihe von Bundesoberbehörden und Bundesinstituten im Zuständigkeitsbereich des Ministeriums für Gesundheit und Soziale Sicherung (BMGS) sowie des Bundesministeriums für Verbraucherschutz, Ernährung und Landwirtschaft (BMVEL). Die bei der Bekämpfung von Infektionskrankheiten beteiligten Bundesministerien, Bundesoberbehörden und Bundesinstitute sollen im Folgenden kurz vorgestellt werden.

\section{Strukturen auf Bundesebene}

\section{Bundesministerium für Verbraucherschutz, Ernährung und Landwirtschaft}

Vorsorgender Verbraucherschutz, Qualitätssicherung sowie umwelt- und tiergerechte Erzeugung sind die 3 Hauptziele der Verbraucher-, Ernährungs- und Agrarpolitik des Bundes. Um diese Ziele besser umzusetzen, wurde im Jahr 2001 das Bundesministerium für Ernährung, Landwirtschaft und Forsten (BML) zum Bundesministerium für Verbraucherschutz, Ernährung und Landwirtschaft (BMVEL) umgebildet. Dem neuen Ministerium wurden aus dem Geschäftsbereich des Bundesministeriums für Gesundheit die Zuständigkeiten für den Verbraucherschutz sowie aus dem Bundesministerium für Wirtschaft und Technologie die Zuständigkeit für die Verbraucherpolitik übertragen.

Zum Geschäftsbereich des Ministeriums zählen - neben dem neu hinzugekommenen Bundesinstitut für Risikobewertung (BfR) und dem BVL - unter anderem das Bundessortenamt, 7 Bundesforschungsanstalten sowie die Zentralstelle für Agrardokumentation und -information. Darüber hinaus hat das Ministerium die Aufsicht über 5 Anstalten des öffentlichen Rechts, darunter die Bundesanstalt für Landwirtschaft und Ernährung [5].

\section{Das Bundesinstitut für Risikobewertung}

Das Bundesinstitut für Risikobewertung (BfR) ist neben dem BVL und der Bundesforschungsanstalt für die Viruskrankheiten der Tiere (BFAV) eine der 3 Nachfolgeeinrichtungen des Ende Oktober 2002 aufgelösten Bundesinstituts für gesundheitlichen Verbraucherschutz und Veterinärmedizin (BgVV). Das BfR ist die wissenschaftliche Einrichtung der Bundesrepublik Deutschland, die auf der Grundlage international anerkannter wissenschaftlicher Bewertungskriterien Gutachten und Stellungnahmen zu Fragen der Lebensmittelsicherheit und des gesundheitlichen Verbraucherschutzes erarbeitet. Das BfR formuliert, basierend auf der Analyse der Risiken, Handlungsoptionen zur Risikominderung. Das Institut nimmt hiermit eine wichtige Aufgabe bei der Verbesserung des Verbraucherschutzes und der Lebensmittelsicherheit wahr. Die Bewertungen sollen für die Öffentlichkeit, Wissenschaft und für andere beteiligte oder interessierte Kreise transparent dargestellt und nachvollziehbar sein. Die Bewertungsergebnisse werden - unter Wahrung der Vertraulichkeit geschützter Daten - öffentlich zugänglich gemacht [6].

Das Lebensmittel- und Bedarfsgegenständegesetz (LMBG) definiert Lebensmittel als „Stoffe, die dazu bestimmt sind, in unverändertem, zubereitetem oder verarbeitetem Zustand von Menschen verzehrt zu werden“ (LMBG $\$ 1$ ). Sie können sowohl von Pflanzen als auch vom Tier sowie mit neuen Methoden gewonnen werden. Nach dem LMBG dürfen Lebensmittel nur in Verkehr gebracht werden, wenn sie gesundheitlich unbedenklich sind. Risiken für die Gesundheit können von Stoffen sowie von Mikroorganismen und Parasiten ausgehen. Das BfR bewertet deshalb Lebensmittel hinsichtlich stofflicher und mikrobieller Risiken.

Lebensmittel werden vom BfR außerdem nach ernährungsmedizinischen Kriterien beurteilt. Im Rahmen dieser Beurteilung nimmt die Säuglingsernährung einen bedeutenden Platz ein. Am BfR ist die Nationale Stillkommission angesie- delt, die Empfehlungen zum Stillen und zur Stillförderung erarbeitet und herausgibt, die auch Fragen des Infektionsschutzes berücksichtigen.

Das BfR ist bestrebt, ständig aktuelle Bewertungen von Risiken, die von Lebensmitteln ausgehen können - sei es durch Schwermetalle, Pestizidrückstände, giftige Inhaltsstoffe oder Ähnliches, seien es Risiken, die von neuartigen Technologien der Lebensmittelherstellung ausgehen (Novel Foods) oder mikrobielle Risiken durch Bakterien, Viren, Pilze oder Parasiten - , zu erstellen. Auch die Bovine Spongiforme Enzephalopathie- (BSE-)Erkrankung wird den mikrobiellen Risiken zugeordnet.

In Lebensmitteln können viele Mikroorganismen (Bakterien, Viren, Pilze) und Parasiten, die Krankheiten auslösen, vorkommen. Solche Erreger finden sich sowohl in pflanzlichen Lebensmitteln als auch in Lebensmitteln, die vom Tier stammen. Krankheiten, die vom Tier auf den Menschen übergehen können, bezeichnet man als Zoonosen. Wichtige Erreger derartiger Infektionserkrankungen sind z. B. Salmonellen, E. coli (Fäkalkeime), Mykobakterien, Brucellen, Campylobacter, Listerien, Toxoplasmen, Yersinien, Tollwutviren, Coxiellen, Prionen und verschiedene Parasiten, wie z. B. Trichinen oder Echinokokken. Grundlage für die gesundheitliche Bewertung mikrobieller Risiken in Lebensmitteln ist immer der jeweilige internationale wissenschaftliche Kenntnisstand. Die Abschätzung des gesundheitlichen Risikos, das von einem Erreger ausgeht, ist ein dynamischer Prozess, der fortwährend im Fluss ist. Empfehlungen des BfR werden daher den jeweils vorliegenden neuen Erkenntnissen angepasst. $\mathrm{Zu}$ den Aufgaben des BfR zählt auch die Erforschung von Zoonoseerregern, von Übertragungswegen und der Verbreitung der Zoonosen. Eine weitere Aufgabe ist die Entwicklung alltagstauglicher Diagnosemethoden. Am BfR sind zudem nationale und europäische Referenzlaboratorien für verschiedene Zoonoseerreger angesiedelt.

\section{Das Bundesamt für Verbraucher- schutz und Lebensmittelsicherheit}

Das BVL ist im Zuge der Neuorganisation des gesundheitlichen Verbraucherschutzes mit Wirkung zum 1. Novem- 
Bundesgesundheitsbl - Gesundheitsforsch - Gesundheitsschutz 2005 • 48:963-970

DOI 10.1007/s00103-005-1127-1

C) Springer Medizin Verlag 2005

\section{O. Hamouda}

\section{Die Rolle des Bundes bei der Verhütung und Bekämpfung von Infektionskrankheiten. Strukturen, Zuständigkeiten und Aufgaben - eine Übersicht}

\section{Zusammenfassung}

Die Gesundheitspolitik steht heute und auch in Zukunft vor der Herausforderung, das Gesundheitswesen qualitativ auf einem hohen Stand und gleichzeitig finanzierbar zu halten. Dazu bedarf es eines umfassenden Systems gesundheitlicher Sicherung, das allen Bürgern wirksam und ohne Hindernisse zur Verfügung steht. Wenngleich die Bürger in hohem Maß für die Förderung und den Erhalt ihrer Gesundheit selbst verantwortlich sind, ist es die Aufgabe des Staates, gegen Gefahren Vorsorge zu treffen, die von gefährlichen Krankheitserregern, Produkten oder auch von Umwelteinflüssen ausgehen können. Es gilt, den Schutz gegen bisher bekannte Gefahren auszubauen, neuen Bedrohungen entgegenzutreten und neue wissenschaftliche Erkenntnisse zu berücksichtigen. Prävention, Gesundheitsförderung und Gesundheitsschutz sind wichtige Schlüssel zur Verbesserung des Gesundheitszustands der Bevölkerung sowie zur Senkung der Kosten des Gesundheitssektors und stellen daher eine wichtige Säule im Gesundheitswesen dar. Die Prävention von Infektionskrankheiten und der Infektionsschutz bilden dabei prioritäre Aufgaben in der Fortentwicklung eines leistungsfähigen Gesundheitswesens in Deutschland.

\section{The role of the Federal Government in the prevention and control of infectious diseases. Organizational structures, duties and responsibilities: a short synopsis}

\section{Abstract}

Health policy is faced today with the challenge of keeping the health care system up to a high standard and affordable at the same time. A comprehensive health protection system that is efficient and readily accessible to all is required. Although it is to a great extent the responsibility of every citizen to promote and sustain their own health, it is the duty of the Federal Government to provide for risks that can emanate from pathogens, products or environmental factors.
Protection against all known risks should be improved, new health threats have to be countered and new scientific findings must be considered. Prevention, health promotion and health protection are important key factors to improve health in the general population and to reduce health care costs. The prevention of and protection from infectious diseases are high priority tasks in the further development of an effective health care system in Germany. In order to accom-
Die Bewältigung der genannten Aufgaben bei der Bekämpfung von Infektionskrankheiten erfordert das enge Zusammenwirken einer Reihe von Bundesoberbehörden und Bundesinstituten im Zuständigkeitsbereich des Ministeriums für Gesundheit und Soziale Sicherung sowie des Bundesministerium für Verbraucherschutz, Ernährung und Landwirtschaft.

\section{Schlüsselwörter}

Infektionskrankheiten · Verhütung .

Bekämpfung · Bundesbehörden

plish these tasks in the control of infectious diseases close collaboration between a number of federal agencies and federal institutes under the responsibility of the Ministry of Health and Social Security as well as the Ministry of Consumer Protection, Food and Agriculture has to be ensured.

\section{Keywords}

Infectious diseases · Prevention 
ber 2002 errichtet worden und neben BfR und BFAV eine der 3 Nachfolgeeinrichtungen des BgVV. Wie bereits dargestellt, obliegt dem BfR die Risikobewertung, während das BVL Aufgaben im Bereich des Risikomanagements wahrnimmt. Zu seinen Hauptaufgaben zählen der Betrieb des europäischen Schnellwarnsystems vor gefährlichen Lebensmitteln und Futtermitteln sowie das Krisenmanagement [7].

Die Verbesserung des gesundheitlichen Verbraucherschutzes soll unter anderem durch den bundeseinheitlichen Vollzug der Lebensmittelüberwachung erreicht werden. Das Bundesamt wirkt daher an der Vorbereitung allgemeiner Verwaltungsvorschriften zur Durchführung von Gesetzen im Bereich des gesundheitlichen Verbraucherschutzes und der Lebensmittelsicherheit (LMBG) mit (u. a. zur Durchführung des Lebensmittel- und Bedarfsgegenständegesetzes, Futtermittelgesetzes, Düngemittelgesetzes, Pflanzenschutzgesetzes, Tierschutzgesetzes, Tierseuchengesetzes, Fleischhygienegesetzes). Zusätzlich koordiniert das BVL die Vorbereitung und Begleitung von Überwachungsprogrammen, die durch die Länder durchgeführt werden. Zu diesem Zweck werden BundLänder-Ausschüsse gebildet. Das Bundesamt übernimmt hier die Funktion der Geschäftsstelle.

Auf europäischer Ebene besteht ein Schnellwarnsystem, über das nach einem vorgeschriebenen Verfahren Informationen über bestimmte Produkte, von denen Gefahren für den Verbraucher ausgehen oder ausgehen können, schnellstmöglich an die Mitgliedsstaaten der Europäischen Union (EU) weitergeleitet werden. Zur Sicherstellung der schnellen Informationsweitergabe an die zuständigen Stellen in den Bundesländern übernimmt das Bundesamt die Funktion der nationalen Kontaktstelle für das europäische Schnellwarnsystem im Lebensmittel- und Futtermittelbereich sowie in bestimmten anderen Bereichen der Produktsicherheit.

Dem BVL obliegen auf nationaler Ebene operative Aufgaben des Krisenmanagements (einschließlich Krisenprävention insbesondere Frühbeobachtung und Rückverfolgbarkeit). Es kommuniziert die fachlichen Aspekte der Lebensmittelsicherheit und koordiniert den Austausch diesbezüg- licher Fachinformationen zwischen Bund und Ländern in Krisen und für andere Zwecke zur Vermeidung von Krisen.

Zum BVL gehört weiterhin das Europäische und Nationale Referenzlabor für Rückstände nach der Richtlinie 96/23/EG mit Sitz in Berlin. Es fungiert als nationale Kontaktstelle zur Koordinierung und Unterstützung der Tätigkeiten der Untersuchungsämter der Bundesländer bei Kontrollmaßnahmen von Tieren und tierischen Erzeugnissen auf Rückstände. Die bei der Überwachung gewonnenen Ergebnisse werden durch das Referenzzentrum an die EU übermittelt.

Dem BVL werden nach $\$ 46 \mathrm{~d}$ Abs. 5 LMBG von den zuständigen Länderbehörden die bei der Durchführung des Lebensmittel-Monitorings erhobenen Daten zur Aufbereitung, Zusammenfassung, Dokumentation und Berichterstattung übermittelt. Die Übermittlung erfolgt auf der Grundlage der allgemeinen Verwaltungsvorschrift über die Übermittlung von Daten aus der amtlichen Lebensmittel- und Veterinärüberwachung sowie dem Lebensmittel-Monitoring (AVV-Düb). Es handelt sich hierbei um Daten, die nach Vorgaben verschiedener EG-Richtlinien zu erheben sind.

Das Bundesamt nimmt die Funktion einer öffentlichen Zentralstelle nach Artikel 4 der Richtlinie 96/23/EG, der „Rückstandskontrollrichtlinie“, wahr. Zu den Aufgaben der Zentralstelle gehören die jährliche Erstellung des Nationalen Rückstandskontrollplanes, die Sammlung und Bewertung der nach den Vorgaben der Richtlinie 96/23/EG erzielten Ergebnisse sowie die Weiterleitung und Veröffentlichung dieser Ergebnisse. Das Bundesamt ist Lebensmittelprüfstelle nach $\$ 1$ Abs. 1 Nr. 2 der neuartigen Lebensmittel- und Lebensmittelzutaten-Verordnung für neuartige Lebensmittel- und Lebensmittelzutaten. Die Lebensmittelprüfstelle führt so genannte Erstprüfungen durch. Das Bundesamt ist für die Prüfung der benannten neuartigen Lebensmittel und neuartigen Lebensmittelzutaten zuständig, wenn ein entsprechendes Erzeugnis erstmalig in der Bundesrepublik Deutschland in den Verkehr gebracht werden soll. Das BVL nimmt entsprechende Anträge, die vor dem erstmaligen Inverkehrbringen zu stellen sind, entgegen.
Bundesministerium für Gesundheit und Soziale Sicherung

Zu den im Bereich der Verhütung und Bekämpfung von Infektionskrankheiten relevanten Einrichtungen des BMGS zählen das Paul-Ehrlich-Institut (PEI), das Bundesinstitut für Arzneimittel und Medizinprodukte (BfArM), die Bundeszentrale für gesundheitliche Aufklärung (BZgA) sowie das Robert Koch-Institut (RKI).

\section{Paul-Ehrlich-Institut}

Am 1. November 1972 wurde das PEI mit dem „Gesetz zur Errichtung eines Bundesamtes für Sera und Impfstoffe " zu einer selbstständigen Bundesoberbehörde. Zu seinen Aufgaben zählen die staatliche Zulassung und Chargenprüfung (immun)biologischer Arzneimittel wie Impfstoffe und Sera, die in Deutschland in den Verkehr gebracht werden sollen, sowie die prüfungsbegleitende Forschung [8].

Die heutigen Aufgaben des PEIs gemäß Arzneimittelgesetz (AMG) umfassen neben der Zulassung und Chargenprüfung immunbiologischer und hämatologischer Arzneimittel auch die Pharmakovigilanz (Arzneimittelsicherheit) und die Teilnahme seiner Experten an Inspektionen im Zusammenhang mit diesen Präparaten, die von den zuständigen Behörden der Bundesländer durchgeführt werden. Zu den Arzneimitteln, für die das PEI verantwortlich ist, gehören Impfstoffe für Mensch und Tier, Allergene, Immunglobuline, Sera, monoklonale Antikörper (die gemäß Arzneimittelgesetz wie Sera behandelt werden) und Blutprodukte (einschließlich rekombinante Blutprodukte). Im Gegensatz zur Situation in den meisten anderen Mitgliedsstaaten der Europäischen Union ist das PEI zusätzlich auch für die Zulassung von Blutkomponenten zur Transfusion zuständig. Dies bedeutet, dass jede Blutspendeeinrichtung, die Konzentrate von Erythrozyten und Thrombozyten produziert, eine Zulassung beim PEI beantragen muss.

Jedes Fertigarzneimittel (Arzneimittel, die im Voraus hergestellt und in bestimmten Packungsgrößen an den Verbraucher abgegeben werden) muss ein Zulassungsverfahren durchlaufen, bevor es in Deutschland in den Verkehr gebracht werden darf. Dies gilt für pharmazeutische 
Präparate genauso wie für biologische Präparate. In diesem Zulassungsverfahren müssen Qualität, Wirksamkeit und Unbedenklichkeit sowie Umweltverträglichkeit belegt werden.

1994 wurde dem PEI mit der Zuständigkeit für Blut und Blutprodukte eine neue, verantwortungsvolle Aufgabe übertragen. Diese Präparate werden, wie andere biologische Arzneimittel auch, einer intensiven experimentellen Überprüfung und Kontrolle unterzogen. Im Gegensatz zu den pharmazeutischen Arzneimitteln unterliegen biologische Präparate noch der Chargenprüfungspflicht. Das bedeutet, dass nach erfolgter Zulassung jede einzelne Charge des Arzneimittels durch das PEI freigegeben werden muss, bevor sie verkauft werden darf.

Am Beispiel eines Präparates, das aus menschlichem Blut oder Plasma hergestellt wurde, bedeutet dies - neben vielen anderen Kriterien, die überprüft werden beispielsweise:

- Es muss in der Dokumentation nachgewiesen werden, dass jede Einzelspende, die in einem Plasmapool eingebracht wurde, auf HIV, Hepatitis-Bund Hepatitis-C-Viren getestet wurde und frei von diesen ist.

- Der Herstellungsgang muss die erforderlichen Schritte zur Virusinaktivierung enthalten, und deren Erfolg muss überprüft werden.

Die Wirksamkeit dieser Verfahren muss wiederum durch so genannte Virusvalidierungsstudien belegt werden. Solche Inaktivierungsverfahren sind notwendig, um Viren abzutöten, die dem Suchtest evtl. entgangen sind, weil vom Immunsystem noch nicht ausreichend viele Antikörper gebildet wurden, und daher der Nachweis durch einen Test nicht erfolgen kann (diagnostisches Fenster). Das ist der Fall, wenn die Infektion noch sehr frisch ist. Zum anderen führt das PEI selbst Tests an Proben aus dieser Charge durch, um beispielsweise die Wirksamkeit zu überprüfen oder um sicherzustellen, dass keine Fieber erregenden Stoffe das Präparat verunreinigen. Erst wenn das PEI bescheinigt hat, dass alle Kriterien erfüllt sind und eine ordnungsgemäße Dokumentation vorliegt, darf ein solches Präparat verkauft werden. Die
Chargenprüfungspflicht wurde für diese Arzneimittel schrittweise eingeführt. Mit Wirkung vom 1. Januar 1996 sind alle Blutzubereitungen der staatlichen Chargenprüfungspflicht unterstellt.

Im Rahmen der Spontanerfassung sollen dem PEI unerwünschte Arzneimittelwirkungen - einschließlich durch Arzneimittel übertragene Infektionen - der Präparate aus dem Verantwortungsbereich des Instituts gemeldet werden. Dies kann durch den behandelnden Arzt, die Arzneimittelkommission der Bundesärztekammer oder den pharmazeutischen Unternehmer geschehen. Der pharmazeutische Unternehmer ist gesetzlich verpflichtet, den Verdacht einer schwerwiegenden $\mathrm{Ne}$ benwirkung innerhalb von 15 Tagen nach Bekanntwerden an das PEI zu melden. Für Verdachtsfälle schwerwiegender Nebenwirkungen nach Impfung besteht seit Inkrafttreten des IfSG ( $\$ 6$, Abs. 1, Satz 3) eine gesetzliche Verpflichtung. Im Referat Arzneimittelsicherheit des PEIs werden diese Meldungen erfasst und ausgewertet. Ergeben sich dabei Hinweise auf schädliche Wirkungen durch ein Präparat, kann das PEI ein Stufenplanverfahren einleiten, um die Unbedenklichkeit des Arzneimittels neu zu beurteilen. Stellt sich im Verlauf dieses Stufenplanverfahrens ein begründeter Verdacht auf Bedenklichkeit heraus, kann das Institut verschiedene Maßnahmen anordnen. Diese reichen von Auflagen (beispielsweise Aufnahme eines Warnhinweises in den Beipackzettel oder Aufnahme einer bekannten, aber tolerierbaren Nebenwirkung in den Beipackzettel) über das Ruhen der Zulassung des Präparates bis hin zum Widerruf der Zulassung und dem Rückruf einzelner Chargen oder des ganzen Präparates.

Zu den Amtsaufgaben des PEIs gehört auch, Inspektionen bei pharmazeutischen Unternehmern durchzuführen, die Blut, Blutprodukte, Sera oder Impfstoffe herstellen, bzw. die Unterstützung der Landesbehörden bei der Überwachung.

\section{Das Bundesinstitut für Arzneimittel und Medizinprodukte}

Das BfArM ist eine selbstständige Bundesoberbehörde im Geschäftsbereich des BMGS. Hervorgegangen ist es aus dem am 1. Juli 1975 gegründeten Institut für Arzneimittel des aufgelösten Bundesgesundheits- amtes. Ziel der Arbeit des BfArM ist die Abwehr von Gesundheitsgefahren durch die kontinuierliche Verbesserung der Sicherheit von Arzneimitteln und die Risikoüberwachung von Medizinprodukten sowie die Überwachung des Betäubungsmittel- und Grundstoffverkehrs [9].

Neben der Zulassung von Fertigarzneimitteln auf der Grundlage des Arzneimittelgesetzes bei der der Nachweis der Wirksamkeit, die Unbedenklichkeit und die angemessene pharmazeutische Qualität geprüft wird, die den Schwerpunkt der Arbeit des BfArM bildet, zählt auch die Risikoüberwachung bei Arzneimitteln zu seinen Aufgaben. Wenn ein Arzneimittel nach der Zulassung in den Verkehr gebracht und von vielen Patienten angewendet wird, können seltene, zuvor nicht aufgefallene Nebenwirkungen entdeckt werden. Das BfArM sammelt und bewertet entsprechende Berichte von Ärzten und pharmazeutischen Unternehmen. Dies betrifft auch Berichte über Infektionsereignisse. Das BfArM entscheidet dann darüber, ob die Fach- und Gebrauchsinformationen für die entsprechenden Arzneimittel geändert werden müssen, um diesen Risiken Rechnung zu tragen. Erweisen sich die erkannten Nebenwirkungen eines Arzneimittels als so schwerwiegend oder häufig, dass sie den Nutzen übersteigen, nimmt das BfArM die Zulassung auch wieder zurück. Bei seiner Ermittlung, Bewertung und Abwehr arzneimittelbedingter Gefahren steht es in ständigem Austausch mit anderen Behörden der EU und der Weltgesundheitsorganisation (WHO).

Auch im Bereich der Medizinprodukte schließt deren Überwachung Infektionsrisiken mit ein. Medizinprodukte sind Produkte, die insbesondere zum Erkennen und Verhüten, zur Behandlung oder Linderung von Krankheiten oder Verletzungen, zur Untersuchung oder Ersetzung des anatomischen Aufbaus und zur Empfängnisverhütung eingesetzt werden. Hierzu zählen u. a. medizinisch-technische Geräte (z. B. Herzschrittmacher, Röntgengeräte), Implantate, Produkte zur Injektion, Infusion, Transfusion und Dialyse (z. B. Katheter, Spritzen), medizinische Instrumente, Dentalprodukte (z. B. Zahnfüllmaterialien), Verbandstoffe, Sehhilfen, Produkte zur Empfängnisregelung sowie Invitro-Diagnostika. Treten bei der Anwen- 
dung dieser Produkte schwerwiegende Risiken auf, müssen sie dem BfArM von den Herstellern, Betreibern und Anwendern gemeldet werden. Das BfArM ermittelt dann in Zusammenarbeit mit den Herstellern (ggf. unter Beteiligung weiterer Fachkreise) die Ursachen und empfiehlt geeignete Maßnahmen zur Risikoabwehr. Medizinprodukte, die zertifiziert und mit dem CE-Zeichen versehen wurden, sind im gesamten europäischen Wirtschaftsraum verkehrsfähig. Das BfArM arbeitet daher in Fragen der Risikoabwehr mit den zuständigen Behörden im Ausland zusammen.

\section{Die Bundeszentrale für gesundheitliche Aufklärung}

Die BZgA hat das Ziel, die Bereitschaft der Bürger zu einem verantwortungsbewussten, gesundheitsgerechten Verhalten und zur sachgerechten Nutzung des Gesundheitssystems zu fördern. Sie führt dazu bundesweite Aufklärungskampagnen durch und stärkt durch Qualitätssicherungsmaßnahmen die Effektivität und Effizienz gesundheitlicher Aufklärung [10].

Gesundheitserziehung und Gesundheitsförderung sind wichtige Bestandteile des Gesundheitswesens in Deutschland. Seit ihrer Gründung im Jahr 1967 verfolgt die BZgA das Ziel, Gesundheitsrisiken vorzubeugen und gesundheitsfördernde Lebensweisen zu unterstützen. Darüber hinaus ist das Verständnis von Gesundheit und Prävention im Wandel begriffen. Vor diesem Hintergrund ist - als ständiger kommunikativer Prozess - gesundheitliche Aufklärung dem Ziel verpflichtet, eigenverantwortliches Gesundheitshandeln zu ermöglichen.

Gesundheitliche Aufklärung wird in Deutschland als eine übergreifende Daueraufgabe aller staatlichen Ebenen unter Einbindung der Betroffenen durchgeführt. Die BZgA nimmt diese Aufgabe auf Bundesebene als Fachbehörde im Geschäftsbereich des BMGS wahr. Gemäß dem Errichtungserlass vom 20.7.1967 hat sie folgende Aufgaben:

- Erarbeitung von Grundsätzen und Richtlinien für Inhalte und Methoden der praktischen Gesundheitserziehung,

- Ausbildung und Fortbildung der auf dem Gebiet der Gesundheitserzie- hung und -aufklärung tätigen Personen,

- Koordinierung und Verstärkung der gesundheitlichen Aufklärung und Gesundheitserziehung im Bundesgebiet, - Zusammenarbeit mit dem Ausland.

Besondere Kooperationsbeziehungen ergeben sich gemäß dem föderalen Prinzip mit den Bundesländern. Die Länder sind zentrale Partner bei Aufklärungskampagnen mit bundesweiter Bedeutung. Die Kooperation mit Partnern innerhalb des Gesundheitsversorgungssystems (z. B. Ärzte- und Apothekerschaft, Krankenkassen) und außerhalb (z. B. Bildungssystem sowie Arbeits- und Freizeitbereich) ist die Basis für die faktische Umsetzung und Verbreitung gesundheitsfördernder Maßnahmen. Auf internationaler Ebene arbeitet die BZgA insbesondere in den Aktionsprogrammen und Netzwerken der EU. Die BZgA beteiligt sich an den Aktivitäten zur Gesundheitsförderung auch unter dem Aspekt der Qualitätssicherung und an den Programmen zur Bekämpfung von AIDS und Drogen. Somit werden die Möglichkeiten der internationalen Zusammenarbeit zur Verbesserung der Gesundheitserziehung und -förderung in Deutschland genutzt. Ein weiterer wichtiger Partner ist die WHO, für die die BZgA als Kooperationszentrum tätig ist.

Neben der Suchtprävention, Sexualaufklärung und Familienplanung, der Kinder- und Jugendgesundheit, der Prävention ernährungsabhängiger Krankheiten sowie der Durchführung von Informationsund Motivationskampagnen zur Organ-, Blut- und Plasmaspende liegt ein weiterer Arbeitsschwerpunkt der BZgA im Bereich der AIDS-Prävention. Die AIDS-Aufklärungskampagne ist die größte und umfassendste Kampagne zur Gesundheitsprävention in Deutschland. Sie ist modellhaft für eine erfolgreiche, bundesweit öffentlichkeitswirksame Präventionsstrategie. Seit 1987 kombiniert die Kampagne „Gib AIDS keine Chance“ massen- und personalkommunikative Maßnahmen, die sich gezielt an unterschiedliche Gruppen der Bevölkerung wenden. Ihrem Selbstverständnis nach ist die AIDS-Aufklärungskampagne als Dachkampagne angelegt, d. h., sie wirkt übergreifend, komplementär, verstärkend und möglichst nachhal- tig. Hauptziele sind die Verhinderung der Verbreitung von HIV sowie die Schaffung und Stärkung eines gesellschaftlichen Klimas der Solidarität mit und der Nichtausgrenzung von Betroffenen.

Um stabile Präventionseffekte zu erzielen, sind der Aufbau und die Aufrechterhaltung eines auf Dauer ausgerichteten Kommunikationsprozesses notwendig, der die erforderlichen gesellschaftlichen Lernprozesse initiiert und in Gang hält. Die BZgA entwickelte die Kampagne daher mit ineinander verflochtenen Maßnahmeblöcken. Hierzu zählen: massenmediale Angebote (u. a. TV-, Kino- und Radiospots, die „mach's mit“-Kampagne und unterschiedlichste Printmedienangebote), Telefonberatung, personalkommunikative Aktivitäten (z. B. die mobile Großausstellung „LiebesLeben, „Mitmach Parcour" etc.) sowie vielfältige interaktive Angebote im Internet. Die AIDS-Prävention umfasst auch die Prävention anderer sexuell übertragbarer Infektionen und ist je nach Zielgruppe in Sexualpädagogik und Familienplanung mit entsprechend erweitertem Themenkreis eingebettet. Zentrales Element der Kampagnenstruktur ist bis heute die Kooperation und Arbeitsteilung zwischen der BZgA als staatlicher Einrichtung und der Deutschen AIDS-Hilfe (DAH) als Selbsthilfeorganisation. Während sich die BZgA als staatliche Organisation an die Allgemeinbevölkerung wendet, ist die DAH vorrangig für die von HIV und AIDS besonders betroffenen und bedrohten Gruppen zuständig. Die AIDSPrävention der DAH wird von der BZgA finanziert und damit die optimale Synergie beider Bereiche gesichert. Diese Arbeitsteilung ist ein wesentliches Element der erfolgreichen HIV- und AIDS-Prävention in Deutschland. Die BZgA kooperiert ebenfalls eng mit sehr unterschiedlichen Multiplikatorengruppen, wie z. B. den Beratern in Gesundheitsämtern, AIDS-Hilfen und anderen Beratungsstellen, Lehrern, Ärzten, Apothekern, Reiseveranstaltern etc.

\section{Das Robert Koch-Institut}

Das RKI ist die zentrale Einrichtung der Bundesregierung auf dem Gebiet der Krankheitsüberwachung und -prävention und damit auch die zentrale Einrichtung des Bundes auf dem Gebiet der anwen- 
dungs- und maßnahmenorientierten biomedizinischen Forschung. Die Kernaufgaben des RKI sind die Erkennung, Verhütung und Bekämpfung von Krankheiten, insbesondere der Infektionskrankheiten. Als zentrale Einrichtung in der Bundesrepublik Deutschland für das Erkennen von Infektionskrankheiten ist das RKI verantwortlich für die zentrale Zusammenführung der nach dem IfSG erhobenen Meldedaten sowie für die Untersuchung pathogener Viren, Bakterien und Pilze, insbesondere deren Verbreitung, Pathogenese, Immunabwehr und Inaktivierung. Durch Studien zur Ermittlung von Risikofaktoren werden Grundlagen für die Entwicklung gezielter Präventionskonzepte gelegt [11].

Das RKI berät die zuständigen Bundesministerien, insbesondere das BMGS, und wirkt bei der Entwicklung von Normen und Standards mit. Es informiert und berät die Fachöffentlichkeit sowie zunehmend auch die breitere Öffentlichkeit. Im Hinblick auf das Erkennen gesundheitlicher Gefährdungen und Risiken nimmt das RKI eine zentrale „Antennenfunktion“ im Sinne eines Frühwarnsystems wahr. Im Folgenden werden die diesbezüglichen Aufgaben des RKI näher erläutert.

Surveillance. Wesentliche Aufgaben des RKI leiten sich aus dem 2001 in Kraft getretenen IfSG ab. Bei dessen Implementierung hat das RKI als Leitinstitut des Öffentlichen Gesundheitsdienstes (ÖGD) eine weit reichende koordinierende Verantwortung übernommen. Das vom RKI entwickelte Meldesystem erfasst infektionsepidemiologische Daten zur Überwachung der Situation übertragbarer Krankheiten in Deutschland. Die Qualität des IfSG und dessen engagierte Umsetzung in der Bundesrepublik Deutschland ist international anerkannt und bietet durch die vorhandenen Daten und deren Struktur eine optimale Forschungsressource zur Analyse der Ausbreitung und Auswirkungen von Infektionskrankheiten sowie entsprechender Präventionsstrategien. Das deutsche Meldesystem und die angewandten Instrumente der Surveillance können sich zu den führenden ihrer Art in Europa zählen.

Vor dem Hintergrund dieser Aufgaben ergeben sich für das RKI unter anderem Forschungsarbeiten zu folgenden Aspekten:
- Evaluierung der Qualität der Überwachung meldepflichtiger Krankheiten,

- Methodenentwicklung zur Früherkennung von Infektionsgefahren,

- Studien zur Erfassung der Durchimpfung der Bevölkerung,

- Analyse der Verbreitung von und der Risikofaktoren für Tuberkulose (insbesondere multiresistente Tuberkulose),

- Erfassung und Analyse von Infektionsmarkern bei Blutspendern zur Erhöhung der Sicherheit von Blut und Blutprodukten,

- HIV/AIDS und andere sexuell oder durch Blut übertragbare Infektionen sowie

— importierte Infektionen.

Die Erfahrungen aus der Bewertung dieser Daten bieten darüber hinaus Ansatzpunkte für die Entwicklung neuer epidemiologischer Instrumente insbesondere im Bereich der molekularen Epidemiologie und als Basis für Frühwarnsysteme, wie z. B. Sentinel-Netzwerke und Instrumente zur syndromischen Surveillance.

Nach dem Transfusionsgesetz (TFG) werden dem RKI vierteljährlich die Daten zu den Spende- und Untersuchungszahlen sowie die Anzahl bestätigter HIV-, Hepatitis-B-, Hepatitis-C- und Syphilis-Infektionen unter Blutspendern aus allen Blutund Plasmaspendezentren in Deutschland gemeldet. Diese Daten, aufgeschlüsselt nach Alter, Geschlecht und weiteren Parametern, ermöglichen eine zeitnahe Beobachtung und Bewertung von Trends im Auftreten relevanter Infektionen in der Blutspenderpopulation. Die im $\$ 22$ TFG geforderten Daten dienen einer genauen Übersicht über die Prävalenz und Inzidenz der Infektionsmarker im Blut- und Plasmaspendewesen. Hieraus lassen sich wichtige Erkenntnisse über die Sicherheit der Spenderkollektive gewinnen [12].

Das RKI hat zwischenzeitlich auch zentrale Verantwortung für die Prävention, Erkennung und Schadensbegrenzung bei Angriffen oder Anschlägen mit biologischen Agenzien übernommen. Nach dem 11. September 2001 und den Milzbrandanschlägen in den USA wurde im RKI das Zentrum für Biologische Sicherheit (ZBS) eingerichtet, dem die Zentrale Informationsstelle des Bundes für Biologische Sicherheit (IBBS) zugeordnet ist. Die IBBS be- rät politische und andere Entscheidungsträger, die Fachöffentlichkeit sowie interessierte Öffentlichkeit zu Fragen der biomedizinischen Sicherheit. Im ZBS wurde eine umfangreiche Erregerdiagnostik zur Analyse verdächtiger Proben auf- und ausgebaut (s. auch den Beitrag von R. Fock et al. in diesem Heft).

Infektionsprävention. Ein wichtiger Bestandteil der Infektionsprävention ist die Entwicklung und Durchführung epidemiologischer Studien und Sentinels zur Ermittlung von Risikofaktoren und -gruppen für übertragbare Krankheiten [13]. Ausgehend von den in diesen Untersuchungen erhobenen Befunden sowie den Daten zur Surveillance der meldepflichtigen Infektionskrankheiten und Infektionserreger werden gezielt Präventionsmaßnahmen zu HIV/ AIDS und anderen sexuell oder durch Blut übertragbaren Infektionen, zu Zoonosen, gastroenterologischen und importierten Erkrankungen sowie Impfstrategien entwickelt und evaluiert. Darüber hinaus sollen Studien zur Beschreibung der Auswirkungen (Krankheitslast, ökonomische Auswirkungen) übertragbarer Krankheiten im Hinblick auf Morbidität, Mortalität und Kosten konzipiert und durchgeführt sowie entsprechende Modelle entwickelt werden.

Ein wichtiger Forschungsschwerpunkt für die Verhütung und Bekämpfung von Infektionskrankheiten ist die Infektionsprävention. Wie in anderen Industrienationen zählen die Krankenhausinfektionen auch in Deutschland zu den häufigsten Infektionen. Ziel ist es, diese nosokomialen Infektionen sowie die entsprechenden Erreger mit spezifischen Resistenzen sicher und frühzeitig zu erkennen und deren Weiterverbreitung zu verhindern. Es werden daher Untersuchungen zur Inaktivierung von Krankheitserregern, einschließlich Prionen, sowie die Prüfung der Wirksamkeit von Mitteln und Verfahren zur Desinfektion und Sterilisation durchgeführt. Das Erkennen und Überwachen von Chemo- und Antibiotika-resistenten Erregern ist nicht nur für die Behandlung von Patienten von großer Bedeutung. Vor dem Hintergrund der Zunahme multiresistenter Erreger insbesondere im Krankenhausbereich entstehen auch erhebliche zusätzliche Kosten $u$. a. durch die Zahl schwer behandelbarer oder letal verlaufender Infektionen. 
Ausbruchsuntersuchungen. Die Untersuchung von Infektionsausbrüchen dient einerseits der konkreten Bekämpfung einer Bedrohung, bietet aber andererseits auch die Gelegenheit, durch Studien im Rahmen der Ausbruchsuntersuchung neue Erkenntnisse über Vehikel, Übertragungswege, besondere Risikogruppen usw. zu gewinnen. Die Durchführung solcher Studien in Zusammenarbeit mit Gesundheitsämtern, Landesbehörden, Nationalen Referenzzentren und Universitäten ist ein Schwerpunkt der Infektionsepidemiologie am RKI (s. auch den Beitrag von A. Ammon in diesem Heft).

Nationale Referenzzentren und Konsiliarlabore. Im Rahmen der Neustrukturierung der Infektionsepidemiologie in Deutschland werden seit 1995 Nationale Referenzzentren (NRZ) zur Überwachung wichtiger Infektionserreger benannt und durch das BMGS berufen. Die Berufung erfolgt jeweils für eine 3-jährige Periode in Abstimmung mit Vertretern des RKI, der Kommission Infektionsepidemiologie und den medizinisch-wissenschaftlichen Fachgesellschaften. Der Entscheidung über die zu berufenden Referenzzentren liegen Überlegungen zur epidemiologischen Relevanz von Erregern, zur Spezialdiagnostik, aber auch Fragen zur Resistenz und zu Maßnahmen des Infektionsschutzes zugrunde [14].

Masern und Poliomyelitis sollen weltweit ausgerottet werden. Die entsprechenden NRZ am RKI sind durch die WHO als europäische Referenzeinrichtungen in die Eradikationsprogramme eingebunden.

Die Daten des Influenzareferenzlabors leisten einen wichtigen Beitrag für die Festlegung des jährlich zu aktualisierenden Impfstoffs durch die WHO und sind Voraussetzung, um die Zirkulation von Virusvarianten zu charakterisieren, die Effektivität des Impfstoffs zu evaluieren und eine Antigendrift oder -shift zu identifizieren (zur Influenzapandemieplanung s. den Beitrag von W.H. Haas in diesem Heft).

Darüber hinaus ergänzen über 50 Konsiliarlaboratorien, die ihr Beratungsangebot ehrenamtlich leisten, das Netz der NRZs und gewährleisten, dass für ein möglichst breites Spektrum von Krankheitserregern fachlicher Rat vorgehalten wird (s. auch den Beitrag von G. Laude und A. Ammon in diesem Heft).
Kommissionen und Arbeitskreise. Kommissionen und Arbeitskreise am RKI, wie z. B. die Ständige Impfkommission (STIKO), der Arbeitskreis Blut oder die Kommission für Krankenhaushygiene und Infektionsprävention, geben Empfehlungen zu medizinischen Problemen und Entwicklungen, z. B. zum Schutz vor und Umgang mit Infektionserregern oder infektiösen $\mathrm{Ma}$ terialien.

\section{Fazit}

Insgesamt ist es in Deutschland gelungen, ein effizientes Netzwerk von Einrichtungen auf Bundesebene aufzubauen, das den Anforderungen an eine moderne Infektionsprävention und Infektionskontrolle gewachsen ist. Die je nach den Erfordernissen notwendigen Kooperationen sowohl zwischen den verschiedenen Bundesinstituten und Bundesbehörden als auch zwischen den Einrichtungen des Bundes, der Länder und der Kommunen haben sich in den letzten Jahren weiter deutlich intensiviert. Gemeinsam durchgeführte Fortbildungsveranstaltungen und zahlreiche instituts- und behördenübergreifende Kontakte auf Arbeitsebene tragen dazu bei, die etablierten Netzwerke zu festigen und auszubauen. Somit scheint Deutschland auch für die wachsenden Anforderungen im Hinblick auf eine europaweite Verhütung und Bekämpfung von Infektionskrankheiten gut gerüstet zu sein.

\section{Korrespondierender Autor}

\section{Dr. O. Hamouda}

Abteilung für Infektionsepidemiologie,

Robert Koch-Institut,

Postfach 650261, 13302 Berlin

E-Mail:HamoudaO@rki.de

\section{Literatur}

1. Bales S, Baumann HG, Schnitzler N (2003) Infektionsschutzgesetz, Kommentar und Vorschriftensammlung. 2. Auflage. W. Kohlhammer, Stuttgart

2. Krause G, Altmann D, Claus H et al. (2003) Erste Bilanz des neuen Systems zur Überwachung meldepflichtiger Krankheiten nach dem Infektionsschutzgesetz. Gesundheitswesen 65 [Suppl 1]:8-12

3. Krause G (2004) Die Erfahrung im öffentlichen Gesundheitsdienst bei der Umsetzung des neuen Meldewesens nach dem Infektionsschutzgesetz. Gesundheitswesen 66(8-9):522-527
4. Benzler J, Krause $G$ und die Mitarbeiter der Abt. für Infektionsepidemiologie (2004) Zur Ausgabe 2004 der Falldefinitionen für die Surveillance meldepflichtiger Infektionskrankheiten in Deutschland. Bundesgesundhbl Gesundheitsforsch Gesundheitsschutz 47(2):141-146

5. http://www.verbraucherministerium.de/

6. http://www.bfr.bund.de/

7. http://www.bvl.bund.de/

8. http://www.pei.de/

9. http://www.bfarm.de/

10. http://www.bzga.de/

11. http://www.rki.de/

12. Offergeld R, Ritter S, Faensen D, Hamouda O (2004) Bericht des Robert Koch-Instituts zu den Meldungen nach § 22 Transfusionsgesetz für die Jahre 2001 und 2002. Bundesgesundheitsbl Gesundheitsforsch Gesundheitsschutz 47:1216-1229

13. Petersen L, Ammon A, Hamouda O et al. (2000) Developing national epidemiologic capacity to meet the challanges of emerging infections in Germany. Emerg Infect Dis 6(6):576-584

14. RKI (2002) Nationale Referenzzentren und Konsiliarlaboratorien. Epidemiol Bull [Sonderausgabe C] 